\title{
In vivo dual-mode full-field optical coherence tomography for differentiation of types of melanocytic nevi
}

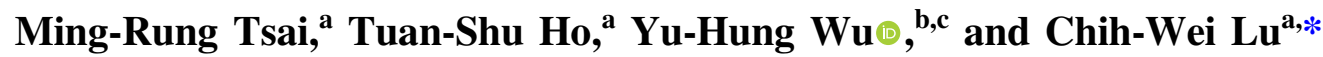 \\ ${ }^{a}$ Apollo Medical Optics, Ltd., Taipei, Taiwan \\ ${ }^{b}$ Mackay Memorial Hospital, Department of Dermatology, Taipei, Taiwan \\ ${ }^{c}$ Mackay Medical College, Department of Medicine, New Taipei City, Taiwan
}

\begin{abstract}
Significance: Melanocytic nevi represent the most common dermal melanocytic lesions in humans. Nevus is typically diagnosed clinically with the naked eye or with dermoscopy. However, it is essential to identify the type of nevus by invasive biopsy for histopathological examination. The use of noninvasive imaging tools can be used to evaluate the types of nevi to reduce unnecessary excisions of benign entities.

Aim: To evaluate the feasibility of using en face and cross-sectional full-field optical coherence tomography (FF-OCT) in differentiation of melanocytic nevi that can facilitate the reduction of unnecessary excisions of benign entities.

Approach: Dual-mode Mirau-type FF-OCT for cross-sectional imaging (B-scan) and en face imaging were used to distinguish the types of nevi.

Results: Although the B-scan reveals the distribution of melanosomes, users can set a specific depth of the en face image to explore the morphology of surrounding skin cells instantly. According to the locations of nevus nests, the different types of nevi, including junction nevus and compound nevus, can be identified using this dual-mode FF-OCT system.

Conclusions: Combining B-scan and en face imaging in vivo FF-OCT enables the examination and navigation of skin tissues in real time and in three dimensions.

(C) The Authors. Published by SPIE under a Creative Commons Attribution 4.0 Unported License. Distribution or reproduction of this work in whole or in part requires full attribution of the original publication, including its DOI. [DOI: 10.1117/1.JBO.26.2.020501]
\end{abstract}

Keywords: melanocytic nevus; optical coherence microscopy; in vivo imaging; cellular resolution.

Paper 200353LR received Nov. 9, 2020; accepted for publication Feb. 3, 2021; published online Feb. 23, 2021.

\section{Introduction}

Melanocytic nevi are common skin lesions in humans. Nevus can be classified as either acquired or congenital according to the onset. They can be further subdivided into junctional, compound, and intradermal nevus based on the histological location and distribution of the proliferating melanocytes in the epidermis and/or dermis. ${ }^{1}$ Several studies have reported that $\sim 25 \%$ to $33 \%$ of cutaneous melanomas arise from nevi. ${ }^{2,3}$ The distinctions between the different histological types of nevi and between nevus and melanoma were based on clinical history, gross morphology, and histopathological features. ${ }^{4,5}$ In clinical practice, nevus can be diagnosed with the naked eye. ${ }^{6}$ The use of dermoscopy by physicians can provide further assessment of clinically atypical melanocytic lesions compared to naked-eye examinations. ${ }^{7}$ The dermoscopy aids in determining the distribution of pigments and blood vessels in the epidermis and papillary dermis, enabling physicians to assess the degree of atypia. However, the gold standard for confirming the type of nevi or melanoma is based on invasive biopsy in histopathological examinations. Newly developed noninvasive imaging tools can provide alternative methods for optical biopsy and enhance the reduction of unnecessary excisions of benign entities.

*Address all correspondence to Chih-Wei Lu, cwlu@mdamo.com 
Recently, several advanced imaging tools have become available for in vivo skin imaging including confocal microscopy ${ }^{8-12}$ and optical coherence tomography (OCT). ${ }^{13-15}$ Confocal microscopy provides unique functions for noninvasive, in vivo assessment of human skin structures using en face imaging with lateral resolution of $1 \mu \mathrm{m} .{ }^{12}$ However, confocal microscopy distinguishes between skin cell layers with difficulty owing to its low axial resolution of $\sim 4 \mu \mathrm{m} .{ }^{12}$ A lateral resolution of 5 to $10 \mu \mathrm{m}$ is more typical in conventional OCT technology. ${ }^{13-16}$ It is challenging to reveal the structure in cellular level. High-resolution OCT can provide an excellent imaging technique for examining cellular characteristics and quantifying the properties of skin structures including full-field OCT $(\mathrm{FF}-\mathrm{OCT})^{17-19}$ and line-field confocal OCT (LC-OCT). ${ }^{20,21}$ Although FF-OCT is a good imaging tool for en face images, the frame rate of cross-sectional imaging (B-scan) is limited by the low brightness of light sources such as halogen lamps and light emitting diodes. ${ }^{18,19}$ LC-OCT is a recently introduced technique for B-scan using a line camera and a supercontinuum light source that provides high-brightness capability for OCT imaging. An en face imaging of LC-OCT could be achieved using a mirror galvanometer for lateral scanning. ${ }^{20}$ However, as compared to one-dimensional scanning, higher en face rates can be achieved by FF-OCT with two-dimensional (2D) parallel detection owing to the commercially available $2 \mathrm{D}$ image sensors with high pixel rates. ${ }^{22,23}$ In addition, an FF-OCT that utilizes a 2D camera can address speckle noise because of spatial compounding. ${ }^{24}$

In this study, we used a high-brightness light source from Ti:sapphire crystal fiber that provides broadband emission for FF-OCT imaging. For the light source, our FF-OCT revealed en face imaging with a lateral resolution of $\sim 1 \mu \mathrm{m}$, and it also increased the frame rate of the B-scan. We present the concept of dual-mode Mirau-type FF-OCT, indicating the B-scan and en face imaging for clinical applications using a simple switch and a flexible probe. As indicated, it is essential to identify the types of nevi for the clinical diagnosis of melanocytic lesions. Therefore, we utilized the dual-mode FF-OCT system to obtain images of normal skin and two types of nevi in the same volunteer for clinical evaluation. Our system can switch between B-scan and en face imaging modes by simply clicking a button that enables clinicians to efficiently explore the nevus. The B-scan images show the distribution of melanosomes and users can set specific depths of the en face images to explore the morphology of the surrounding skin cells instantly. According to the location of the nevus nest, nevus, such as junction nevus, and compound nevus can be identified. Combining B-scan and en face imaging, in vivo FF-OCT enables the examination and navigation of skin tissues in real time and in three dimensions.

\section{Methods}

In this system (ApolloVue S100, Apollo Medical Optics, Ltd., Taipei, Taiwan), we used a glassclad Ti:sapphire crystal fiber to generate broadband amplified spontaneous emission as a light source. $^{25-27}$ The Ti:sapphire crystal fiber was pumped by two 520-nm laser diodes. The center wavelength and bandwidth are 780 and $150 \mathrm{~nm}$, respectively, yielding an OCT system with a high axial resolution of $1.3 \mu \mathrm{m} .{ }^{28}$ The power of the light source was $\sim 20 \mathrm{~mW}$ and the power on the patient was about $5 \mathrm{~mW}$. For the safety of the system, the light source was test according to both IEC 60825-1 and IEC 62471. The system was Federal Drug Administration (FDA)approved as a class II medical device. The bright continuous-wave emission of the crystal fiber source is significantly advantageous for clinical applications compared to pulsed light sources owing to the signal-to-noise ratio (SNR) of FF-OCT being proportional to the average power of the light source. ${ }^{28,29}$

A Mirau-type OCT configuration was adopted to minimize external disturbances such as environmental vibration and sample movement, as shown in Fig. 1(a). The broadband light was delivered into a multimode fiber and reflected by a polarizing cubic beam splitter. The Mirau objective was mounted on a piezoelectric (PZT) stage and the total travelling range of the PZT with open-loop control is $400 \mu \mathrm{m}$. The B-scan and en face images can be obtained by demodulating the interference signals acquired during PZT scans. ${ }^{28}$ The illumination module was integrated with a motorized slider to switch different lens modules to generate strip-field and wide-field illumination for B-scan mode and en face imaging mode, respectively. ${ }^{30}$ The Mirau 
(a)

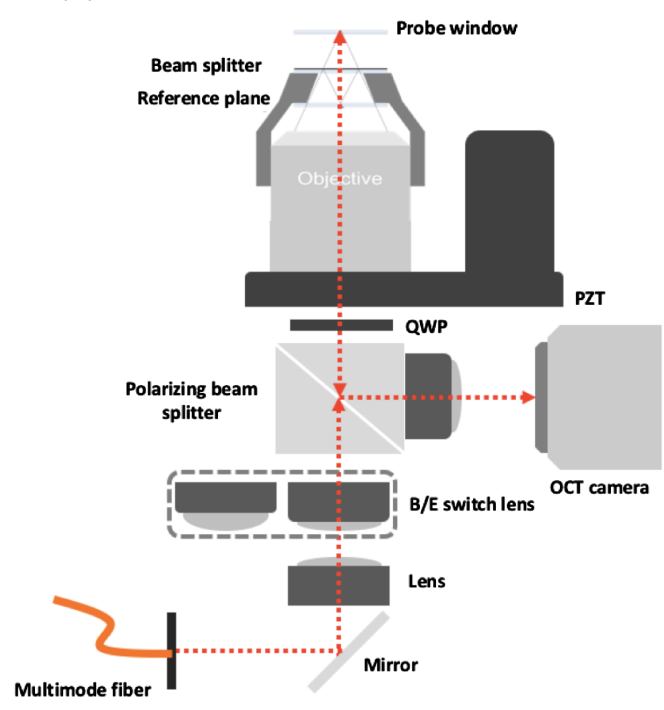

(a)

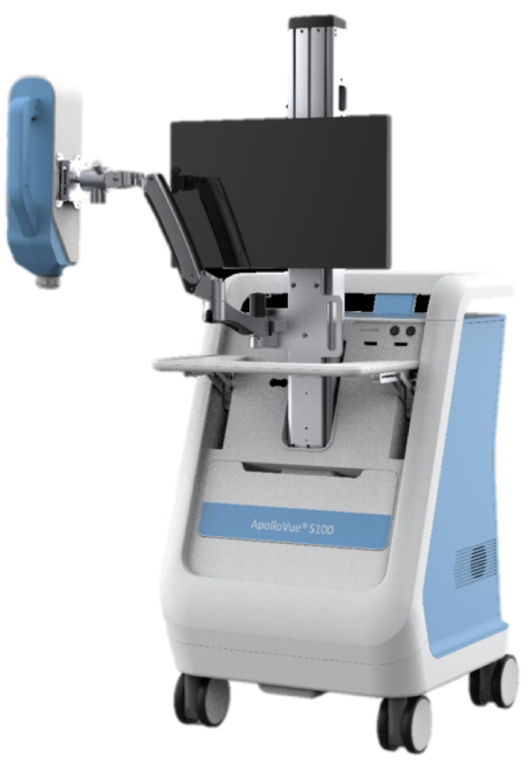

Fig. 1 (a) Schematic of dual-mode Mirau-type FF-OCT system: PZT, piezoelectric stage and QWP, quarter wave plate. (b) Dual-mode Mirau-type FF-OCT system (ApolloVue S100, Apollo Medical Optics, Ltd., Taipei, Taiwan). The system was integrated with a flexible probe that can reach most parts of the skin.

objective was used to illuminate the subject's skin and collect the back-scattered light from the sample and the reference arms. The back-scattered light beams from the sample and reference arms were combined after broadband polarizing cubic beam splitter and projected onto a $2 \mathrm{D}$ camera to generate the interferometric signals. A home-made $20 \times$ oil-immersion objective lens (NA: 0.80) was customized to correct the chromatic and spherical aberration introduced by the glass plates (reference plane and beamsplitter) and used to achieve a lateral resolution of $1 \mu \mathrm{m} .{ }^{28}$ To compensate for the chromatic dispersion of the subject, the Mirau objective was oil immersed. The field of views (FOV) of B-scan and en face images were $500 \times 400 \mu \mathrm{m}^{2}$ (imaging depth) and $500 \times 500 \mu \mathrm{m}^{2}$, respectively. The depth for imaging was controlled by the PZT stage. FFOCT with a high-brightness light source, and a 2D camera can provide a frame rate of $0.43 \mathrm{fps}$ in B-scan and $7 \mathrm{fps}$ in en face imaging with cellular resolution. No frame averaging was performed. For clinical usability, the dual-mode FF-OCT system was integrated with a flexible probe that can reach most parts of the skin, as shown in Fig. 1(b). The probe was mounted on a swing arm that is flexible to rotate $360 \mathrm{deg}$ in the sagittal plane and $180 \mathrm{deg}$ in both the frontal and horizontal planes.

To demonstrate the capability for clinical applications using the in vivo dual-mode OCT system, we obtained images of a normal skin and two melanocytic nevi from the same volunteer (37-year-old, female) to observe the features of images. Nevus 1 and nevus 2 are on the right forearm and the right leg of the volunteer, respectively. The system also provided guiding images using an embedded video dermoscope. The use of guiding images can aid users to navigate observed subjects. Therefore, we used the guiding image to note the skin area of interest and switch to the FF-OCT mode to obtain the OCT images. From the features of images, the normal skin and nevus types can be identified.

\section{Results and Discussion}

A Mirau-type FF-OCT with a $\mathrm{Ce}^{3+}$ :YAG crystal fiber as a light source for obtaining images of in vivo skin has been reported. ${ }^{28}$ The imaging depth using $\mathrm{Ce}^{3+}$ :YAG crystal fiber is $\sim 100 \mu \mathrm{m}$ owing to its wavelength of $560 \mathrm{~nm}$. It is, therefore, difficult to show the structure of the dermis. In this study, we used Ti:sapphire crystal fiber as a light source. FF-OCT using Ti:sapphire crystal fiber with a wavelength of $780 \mathrm{~nm}$ can provide greater imaging depth. 

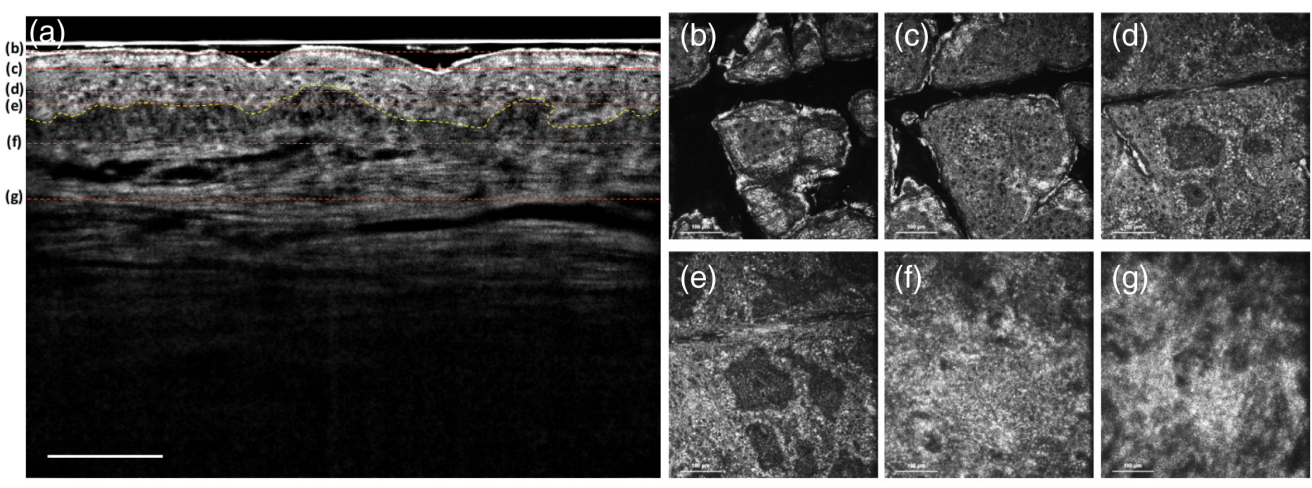

Fig. 2 Normal skin: (a) in vivo FF-OCT B-scan image. The dashed yellow line presents the DEJ. (b)-(g) In vivo FF-OCT en face images at imaging depths of 30, 45, 65, 75, 110, and $160 \mu \mathrm{m}$, respectively. The red dashed lines in (a) show the different depths of the en face images from (b)-(g). From (b)-(e), the different layers of epidermis including the stratum corneum, stratum granulosum, stratum spinosum, and stratum basale can be obtained. (f) and (g) The papillary dermis and reticular dermis, respectively, which have different structures of collagen fibers. Scale bar: $100 \mu \mathrm{m}$.

Figure 2(a) shows the in vivo FF-OCT B-scan image of the normal skin on the forearm, and the imaging depth that can be achieved is over $250 \mu \mathrm{m}$. The B-scan image of human skin shows a vertical image including the epidermis, dermis, and dermal-epidermal junction (DEJ) with cellular resolution simultaneously. The dashed yellow line presents the DEJ, that is the region of skin between the epidermis and dermis. ${ }^{31}$ Our system yielded the en face images shown in Figs. 2(b)-2(g) at imaging depths of 30, 45, 65, 75, 110, and $160 \mu \mathrm{m}$, respectively. The different morphologies of keratinocytes are shown in Figs. 2(b)-2(e), including the stratum corneum, stratum granulosum, stratum spinosum, and stratum basale. The nuclei of keratinocytes were resolved in the epidermis and appeared as black holes in the images. Figures 2(f) and 2(g) show the papillary dermis and reticular dermis, respectively, which have different structures of collagen fibers.

Nevus 1 was on the right forearm of the volunteer. Dermoscopy evaluation of nevus 1 was performed with a digital dermoscope (DermLite Cam, DermLite) and an embedded video dermoscope (built-in S100 system), as shown in Figs. 3(a) and 3(b), respectively. The dual-mode FF-OCT images were obtained from the nevus shown in Figs. 3(c)-3(f). The yellow line in Fig. 3(b) represents the section region of the B-scan in Fig. 3(c). The B-scan image of nevus 1 shows the distribution of melanosomes near the DEJ, and the yellow arrows show the nevus nests in the DEJ. The depth of the nevus nest can be obtained near $100 \mu \mathrm{m}$. Therefore, we obtained en face images at imaging depths of 90, 110, and $130 \mu \mathrm{m}$, respectively, in Figs. 3(d)-3(f), and the images show the elongation of rete ridges and increased hyper-reflective melanocytes, which are features found in histology. ${ }^{32}$ By combining the features of B-scan and en face images, nevus 1 was considered as a junctional nevus.

Nevus 2 was on the right leg of the volunteer. Figure 4(a) shows the dermoscopy of nevus 2. Different dermoscopic findings, including shape, size, color, and distribution of pigmentation, were determined by comparing dermoscopic images of nevus 1 and nevus 2. An in vivo B-scan image is shown in Fig. 4(b). The dashed and solid yellow arrows show the nevus nests in the basal cell layer and dermis, respectively. The nevus nest in the dermis is at an imaging depth of $120 \mu \mathrm{m}$ from the B-scan image of nevus 2. Therefore, we obtained the en face images of nevus 2 at this imaging depth and the en face image provides the morphology of the nevus nest, as shown in Fig. 4(c). B-scan image indicated the locations of nevus nests both in DEJ and dermis, which is a histological feature of compound nevus. ${ }^{33}$ Nevus 2 was considered to be a compound nevus.

FOV and imaging depth are both important for dermatology applications. The FOV of our FF-OCT is $500 \times 500 \mu \mathrm{m}^{2}$ for en face images. Although many lesions are larger than this size, the entire lesion image could be acquired by means of image stitching under the frame rate of $10 \mathrm{fps}$ for en face imaging. As for the depth, it is known that cancers such as melanoma and 

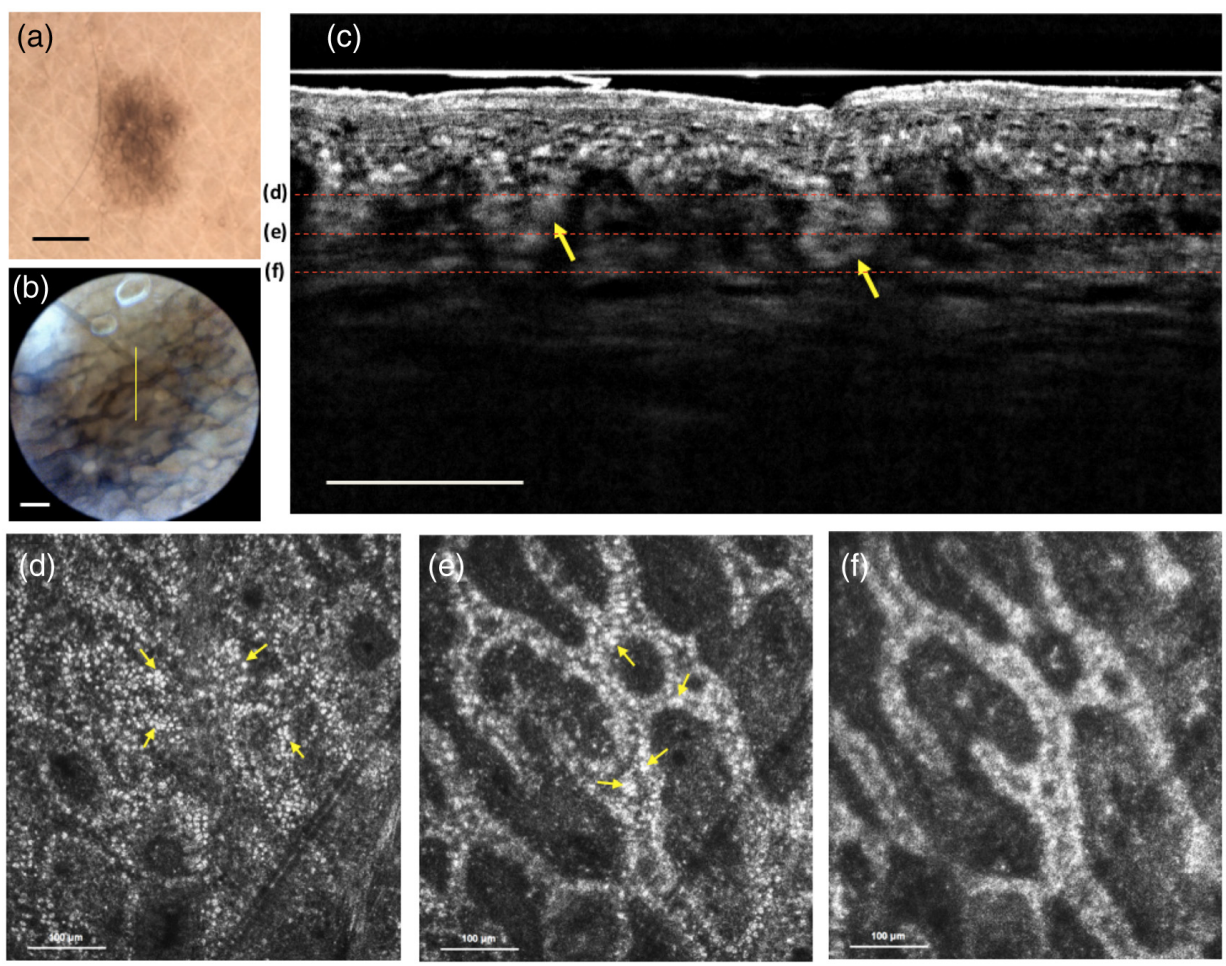

Fig. 3 Junctional nevus: (a) dermoscopy image. Scale bar: $1 \mathrm{~mm}$. (b) The guiding image. The yellow line represents the section region of the B-scan image. Scale bar: $200 \mu \mathrm{m}$. (c) In vivo FF-OCT B-scan image. The yellow arrows show the nevus nest in the DEJ. Scale bar: $100 \mu \mathrm{m}$. (d)-(f) In vivo FF-OCT en face images at imaging depths of 90, 110, and $130 \mu \mathrm{m}$, respectively. The red dashed lines in (c) show the different depths of the en face images from (d)-(f). The images show the elongation of rete ridges and increase hyper-reflective melanocytes. The yellow arrows in (d) and (e) indicate the hyper-reflective melanocytes.
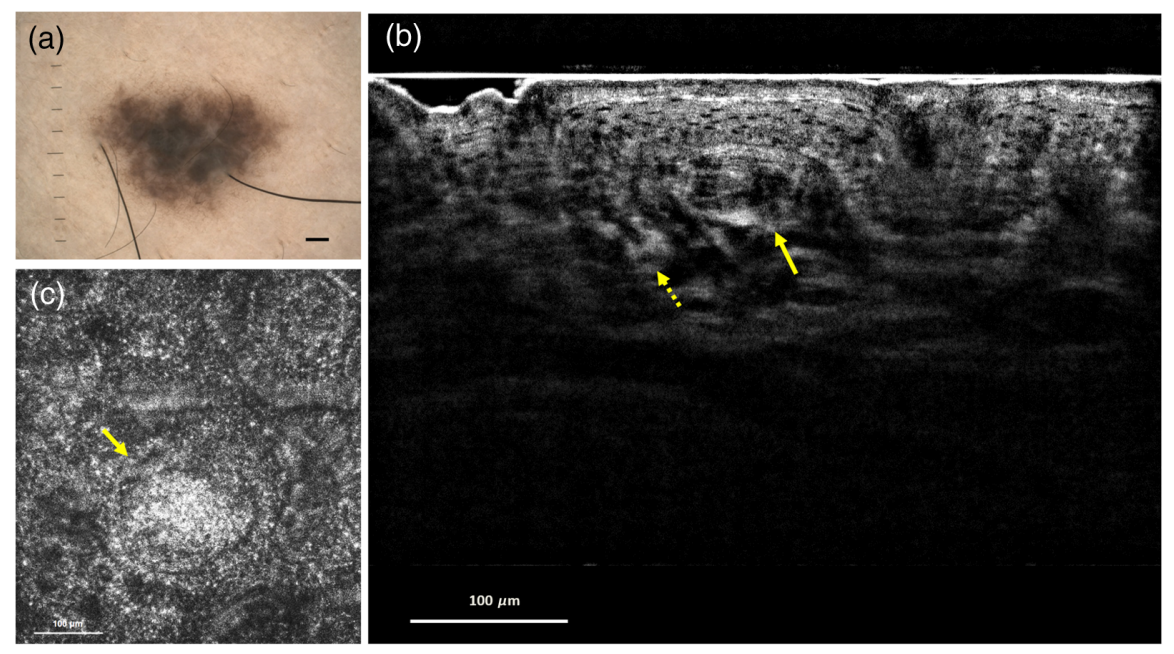

Fig. 4 Compound nevus: (a) dermoscopy image. Scale bar: $1 \mathrm{~mm}$. (b) In vivo FF-OCT B-scan image. The dashed and solid yellow arrows show the nevus nests in the basal cell layer and dermis, respectively. (c) In vivo FF-OCT E-scan image at an imaging depth of $120 \mu \mathrm{m}$. The yellow arrow shows the morphology of the nevus nest in the dermis. 
basal cell carcinoma both originate and spread from DEJ, which is usually within $100 \mu \mathrm{m}$ from the surface. ${ }^{34}$ From Figs. 3(c) and 4(b), it is noted that the DEJ can be clearly revealed in our FF-OCT images. In addition, the nevus nest at the imaging depth of $120 \mu \mathrm{m}$ can be also observed in Fig. 4(c). Therefore, the FOV and imaging depth of the FF-OCT system are adaptable for dermatology applications.

Comparing the light source with the wavelength of $560 \mathrm{~nm},{ }^{28}$ the wavelength of $780 \mathrm{~nm}$ is away from the absorption peaks of melanin and hemoglobin. Therefore, the SNR of the images using the 780-nm wavelength can be higher than that using the 560-nm wavelength. According to the similar measurement method, ${ }^{28}$ the SNR of this system in the epidermis was generally higher than $30 \mathrm{~dB}$.

From the results, combing the B-scan and en face imaging is essential for efficiently showing the structures of nevi. The B-scan view is natural for clinicians, because it is similar to histopathological sections and a significant depth can be obtained for the en face imaging at a specific depth to evaluate the cell morphology. In addition, although common melanocytic nevi are predominantly benign, thus cosmetic removal of facial nevi is a frequent procedure for cosmetic surgeons. ${ }^{35,36}$ The nevus depth is an important parameter that influences the success of laser therapy. ${ }^{37}$ This FF-OCT imaging tool highlights the architecture and distribution of melanocytes and provides useful information for treatment. From the B-scan images of junction nevus and compound nevus in Figs. 3(c) and 4(b), the imaging depths of nevus nests can be obtained, increasing the success rate for nevus removal.

\section{Conclusion}

We present an FF-OCT system that provides imaging of human skin in vivo at cellular resolution in B-scan and en face imaging modes. Compared to the $\mathrm{Ce}^{3+}$ : YAG crystal fiber generating a light source with a wavelength of $560 \mathrm{~nm}$, this system used the Ti:sappire crystal fiber with a 780-nm wavelength light source, which provides a high imaging depth of human skin in vivo. The system adopted a flexible probe, with the possibility for the user to reach most parts of the skin. By clicking a button, the B-scan and en face imaging modes can be switched instantly. These features enable users to explore the skin efficiently.

To address the capability of clinical application, the dual-mode FF-OCT imaging was applied to normal skin and two different nevi for evaluation. Different cell layers in the epidermis, dermis, and clear DEJ can be obtained in the normal skin. From the imaging of the nevus, the B-scans showed the distribution of melanosomes, and users can set specific depths of the en face imaging to explore the morphology of surrounding skin cells instantly. According to the locations of nevus nests, the types of nevi, including junction nevus and compound nevus, can be identified using this dual-mode FF-OCT system. Combining B-scan and en face imaging, in vivo FF-OCT enables the examination and navigation of skin tissues in real time and in three dimensions. The dual-mode FF-OCT technique provides a powerful alternative tool and approach for optical biopsy and reduces unnecessary excisions of benign entities and further clinical applications of skin lesions.

\section{Disclosures}

The authors have no relevant financial interests in this article and no potential conflicts of interest to disclose.

\section{References}

1. R. Happle, "What is a nevus? A proposed definition of a common medical term," Dermatology 191(1), 1-5 (1995).

2. C. Bevona et al., "Cutaneous melanomas associated with nevi," Arch. Dermatol. 139(12), 1620-1624 (2003).

3. W. M. Lin et al., "Outcome of patients with de novo versus nevus-associated melanoma," J. Am. Acad. Dermatol. 72(1), 54-58 (2015). 
4. W. E. Damsky, "Melanocytic nevi and melanoma: unraveling a complex relationship," Oncogene 36(42), 5771-5792 (2017).

5. D. E. Elder, "Precursors to melanoma and their mimics: nevi of special sites," Mod. Pathol. 19, S4-S20 (2006).

6. D. Rigel et al., "The evolution of melanoma diagnosis: 25 years beyond the ABCDs," CA Cancer J. Clin. 60(5), 301-316 (2010).

7. G. A. Holmes et al., "Using dermoscopy to identify melanoma and improve diagnostic discrimination," Fed. Pract. 35(4), S39-S45 (2018).

8. M. Rajadhyaksha et al, "In vivo confocal scanning laser microscopy of human skin II: advances in instrumentation and comparison with histology," J. Invest. Dermatol. 113(3), 293-303 (1999).

9. K. S. Nehal et al., "Skin imaging with reflectance confocal microscopy," Semin. Cutan. Med. Surg. 27(1), 37-43 (2008).

10. P. Calzavara-Pinton et al., "Reflectance confocal microscopy for in vivo skin imaging," Photochem. Photobiol. 84(6), 1421-1430 (2008).

11. V. Ahlgrimm-Siess et al., "Confocal microscopy in skin cancer," Curr. Dermatol. Rep. 7(2), 105-118 (2018).

12. A. Meschieri et al., "Reflectance confocal microscopy: a new tool in skin oncology," Photonics Lasers Med. 2(4), 277-285 (2013).

13. A. Alex et al., "Multispectral in vivo three-dimensional optical coherence tomography of human skin," J. Biomed. Opt. 15(2), 026025 (2010).

14. E. Sattler et al., "Optical coherence tomography in dermatology," J. Biomed. Opt. 18(6), 061224 (2013).

15. M. Ulrich et al., "Dynamic optical coherence tomography in dermatology," Dermatology 232(3), 298-311 (2016).

16. T. Gamblicher et al., "Characterization of benign and malignant melanocytic skin lesions using optical coherence tomography in vivo," J. Am. Acad. Dermatol. 57(4), 629-637 (2007).

17. A. Latrive et al., "In vivo and in situ cellular imaging full-field optical coherence tomography with a rigid endoscopic probe," Biomed. Opt. Express 2(10), 2897-2904 (2011).

18. A. Dubois et al., "High-resolution full-field optical coherence tomography with a Linnik microscope," Appl. Opt. 41(4), 805-812 (2002).

19. A. Dubois et al., "Ultrahigh-resolution full-field optical coherence tomography," Appl. Opt. 43(14), 2874-2883 (2004).

20. J. Ogien et al., "Dual-mode line-field confocal optical coherence tomography for ultrahighresolution vertical and horizontal section imaging of human skin in vivo," Biomed. Opt. Express 11(3), 1327-1335 (2020).

21. A. Dubois et al., "Mirau-based line-field confocal optical coherence tomography," Opt. Express 28(6), 7918-7927 (2020).

22. E. Auksorius et al., "In vivo imaging of the human cornea with high-speed and highresolution Fourier-domain full-field optical coherence tomography," Biomed. Opt. Express 11(5), 2849-2865 (2020).

23. J. Ogien et al., "A compact high-speed full-field optical coherence microscope for highresolution in vivo skin imaging," J. Biophotonics 12(2), e201800208 (2019).

24. M. R. Avanaki et al., "Spatial compounding algorithm for speckle reduction of dynamic focus OCT images," IEEE Photonics Technol. Lett. 25(15), 1439-1442 (2013).

25. K. Y. Hsu et al., "Diode-laser-pumped glass-clad Ti:sapphire crystal fiber based broadband light source," IEEE Photonics Technol. Lett. 24(10), 854-856 (2012).

26. S. C. Wang et al., "Broadband and high-brightness light source: glass-clad Ti:sapphire crystal fiber," Opt. Lett. 40(23), 5594-5597 (2015).

27. S. C. Wang et al., "Laser-diode pumped glass-clad Ti:sapphire crystal fiber laser," Opt. Lett. 41(14), 3217-3220 (2016).

28. C. C. Tsai et al., "Full-depth epidermis tomography using a Mirau-based full-field optical coherence tomography," Biomed. Opt. Express 5(9), 3001-3010 (2014).

29. Y. T. Chen et al., "En Face and cross-sectional corneal tomograms using sub-micron spatial resolution optical coherence tomography," Sci. Rep. 8(1), 14349 (2018). 
30. T. S. Ho et al., "In vivo Mirau-type optical coherence microscopy with symmetrical illumination," Proc. SPIE 11228, 112280X (2020).

31. D.T. Woodley, "Importance of the dermal-epidermal junction and recent advances," Dermatologica 174(1), 1-10 (1987).

32. S. Gundalli et al., "Histopathological spectrum of benign melanocytic nevi-our experience in a tertiary care centre," Our Dermatol. Online 7(1), 21-25 (2016).

33. T. Nathan et al., "A practical approach to the diagnosis of melanocytic lesions," Arch. Pathol. Lab. Med. 143(7), 789-810 (2019).

34. S. Kurugol et al., "Pilot study of semiautomated localization of the dermal/epidermal junction in reflectance confocal microscopy images of skin," J. Biomed. Opt. 16(3), 036005 (2011).

35. J. Niamtu, "Esthetic removal of head and neck nevi and lesions with 4.0-MHz radio-wave surgery: a 30-year experience," J. Oral Maxillofac. 72(6), 1139-1150 (2014).

36. K. Sardana et al., "Optimal management of common acquired melanocytic nevi (moles): current perspectives," Clin. Cosmet. Invest. Dermatol. 7, 89-103 (2014).

37. K. Sardana, "The science, reality, and ethics of treating common acquired melanocytic nevi (moles) with lasers," J. Cutan. Aesthet. Surg. 6(1), 27-29 (2013). 